\title{
Research on logistics handing agv picking route optimization method based on improved genetic algorithm
}

\author{
Juntao $\mathrm{Li}^{1, \mathrm{a}}$, Yan Hao ${ }^{2, \mathrm{~b},{ }^{*}}$, Yinhong Liu ${ }^{3, \mathrm{c}}$ \\ ${ }^{1}$ Beijing Wuzi University, Tongzhou District, Beijing, China \\ ${ }^{2}$ Beijing Wuzi University, Tongzhou District, Beijing, China \\ ${ }^{3}$ Beijing Wuzi University, Tongzhou District, Beijing, China \\ aljtletter@126.com, ${ }^{b}$ hao103@outlook.com, '18810660720@163.com
}

Key words: Logistics handing agv; picking route; genetic algorithm

\begin{abstract}
Establish logistics handing agv operating environment model by using the grid method, and the picking route optimization model based on grid environment model. By analyzing the qualitative factors in agv "goods to person" picking system, present an improved genetic algorithm combining qualitative and quantitative factors, and use the algorithm to solve the picking route optimization model, it can reduce the set of feasible solutions, and get the optimal path which is more fit the actual. Finally through many experiments by using matlab software, the improved genetic algorithm can search a optimized route.
\end{abstract}

\section{Introduction}

With the development of electronic commerce, agv "goods to person" picking system gets more and more attention. In this system, the agv path planning is an important basic work. Over the years, domestic and foreign scholars have achieved fruitful research results on path planning, including grid method, potential field, visibility graph, a * algorithm, $\mathrm{d}^{*}$ algorithm and fuzzy algorithm, neural network algorithm, ant colony algorithm, genetic algorithm and other intelligent algorithms ${ }^{[1]}$.

The purpose of traditional operations research path optimization method is usually to find the shortest path, using the numerical quantitative analysis method to build accurate models and simulate for system is not practical sometimes. Different from the traditional quantitative simulation, qualitative simulation is based on the non-digital methods to process inputting information, modeling, behavior analysis, outputting results. The description of qualitative behaviors can be generated by qualitative modeling. Qualitative simulation can process information in multiple forms, has reasoning and learning ability, preliminary mimic the way of human thinking and the obtained results are easier to understand.

So based on agv grid environmental model, present a genetic algorithm combining qualitative and quantitative factors, and use it to solve the picking route optimization model. It can not only reduce the set of feasible solutions, but also get the optimal solution which is fit the actual. Finally after several simulation experiments, verify the improved algorithm is feasible

\section{Logistics handing agv environmental modeling}

(1) The following assumptions are made when describing the agv runtime environment:

1) Assuming that there is only one agv in the environment, the starting point and the target point of the agv are known. 2) Assuming that there are only static obstacles (movable shelves and picking platform), and every obstacle occupies one grid, and its location is known. 3) Represent the agv working environment in a two-dimensional plane without considering the object height. 4) One agv occupies one grid.

(2) Using the grid method to establish the environmental mold

Using the grid method to map modeling is to partition the agv operating environment into one and one the same size grid, and add passable factor to all the grids, and using the binary method to separately encode the obstacle and channel, and every grid unit also contains a grid 
coordinate $(X, Y)$ and number information ${ }^{[3,4]}$. The coordinate system is shown in Fig. 1, and the environment model is shown in Fig. 3. Based on agv "goods to people" picking practices, agv has four moving directions in the system, forward direction, 90 degrees direction, 180 degrees direction, 270 degrees direction, as shown in Fig. 2.

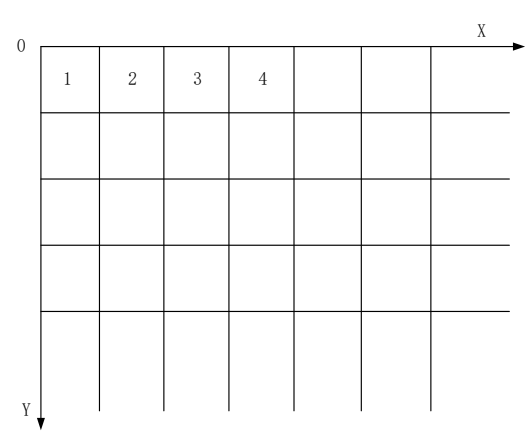

Fig. 1 Grid coordinates and serial number

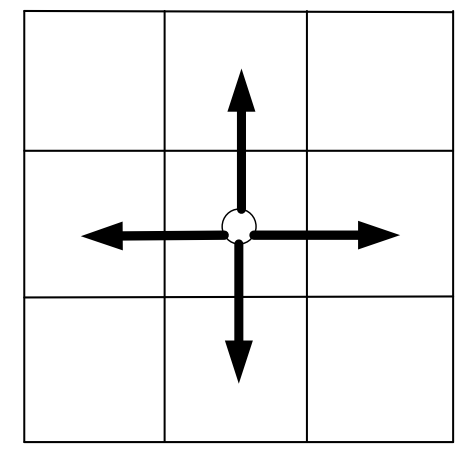

Fig. 2 Agv moving direction

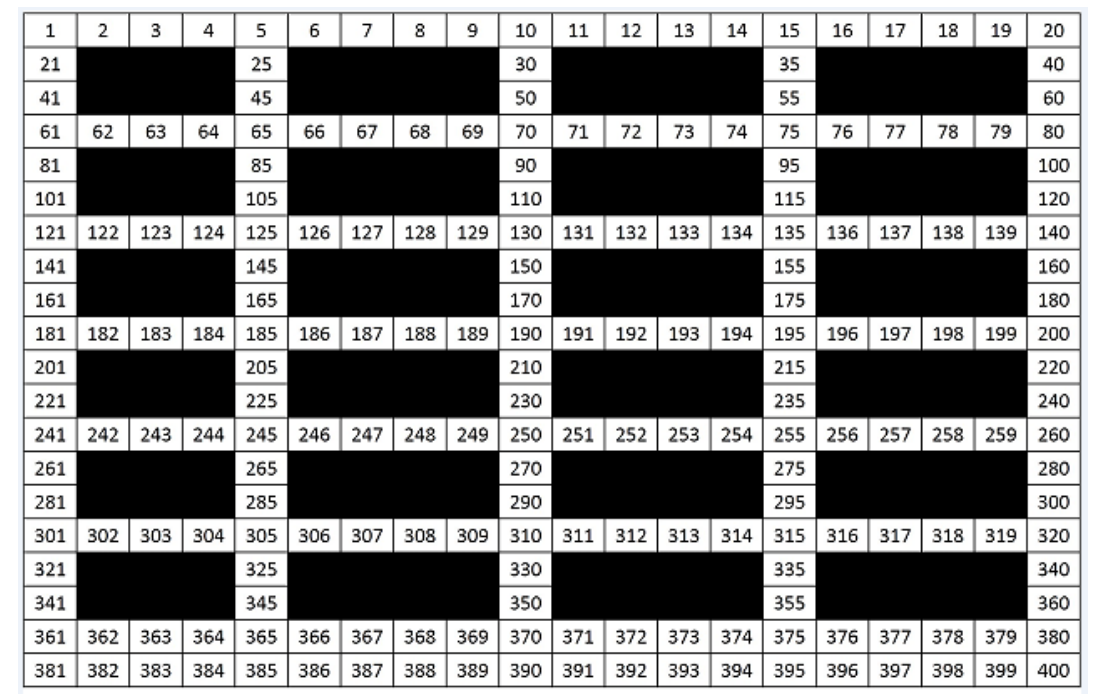

Fig. 3 Schematic diagram of the environmental model

The black grids in the environmental model are obstacle grids in the Fig. 3, and they are coded as 1 ; the white grids are passable, and coded as 0 . Eq. 1 shows the association of two kinds of grid markers.

$$
\begin{cases}T=X+Y * N+1 & \\ X=\bmod (T, N)-1 & \bmod (T, N) \neq 0 \\ X=M-1 & \\ Y=\bmod (T, N) \neq 0\end{cases}
$$

In Eq. 1, abscissa and ordinate respectively represented by $X$ and $Y$, the grid number is $T$, grid row number is $M$, grid column number is $N$.

\section{Logistics handing agv picking route optimization modeling and qualitative factors analysis}

(1) Logistics handing agv picking route optimization modeling

The logistics handing agv in grid environment whether travels from the parking area to the designated area or from designated area to pick station, it all can be described as: agv optimal path planning from the known starting point to the known end point.

Mathematical description: An agv, its starting point and target point is known, reasonably 
arranges of driving route from the starting point $S$ to the target point $T$, and the agv picking route is feasible(agv don't collide with the shelves),and agv walking consumption (route) is minimum.

Suppose that there is a grid map which is $m$ rows and $n$ columns. The initial grid coordinate of agv is $\left(a_{0}, b_{0}\right)$, the target grid coordinate is $\left(a_{m}, b_{n}\right)$. $\mathrm{G}$ is the set of grid coordinate in the map, $A[i][j]$ is the information of the grid $(i, j), R_{a b}$ is the agv grid coordinate $(a, b), D(n)(a, b)$ is the grid coordinate set that the agv walked from $(a, b)$ after $N$ steps, $S$ is all of the feasible solution, $T$ is the optimal solution.

Optimization model are as follows:

$$
\begin{aligned}
& G=\{(i, j) \mid 0<i \leq m, 0<j<n, i, j \in z\} \\
& A[i][j]=\left\{\begin{array}{l}
1,(i, j) \text { is obstacle grid, }(i, j) \in G \\
0,(i, j) \text { is passable grid, }(i, j) \in G
\end{array}\right. \\
& T=\min \{|s|, s \in S\} \\
& \text { Particularly, }|s|=n_{l} \\
& s=\left\{D_{l}(1)\left(a_{0}, b_{0}\right), D_{l}(2)\left(a_{0}, b_{0}\right), \ldots \ldots, D_{l}\left(n_{l}\right)\left(a_{0}, b_{0}\right) \mid D_{l}(k)\left(a_{0}, b_{0}\right) \in G, D_{l}\left(n_{l}\right)\left(a_{0}, b_{0}\right)=\right. \\
& \left.\left(a_{m}, b_{n}\right), k=1,2, \ldots \ldots, n_{l}\right\}
\end{aligned}
$$

(From the starting grid point, after $n_{l}$ steps, arriving at the target grid point)

$$
\begin{aligned}
& \text { If }(s, t)=D_{l}(n-1)(a, b), \\
& D_{l}(n)(a, b)=(x, y),(x, y) \in R_{a b} \\
& A[x][y]=0 \\
& R_{a b}=\{(a+1, b),(a, b+1),(a-1, b),(a, b-1) \mid(a, b) \in S\}
\end{aligned}
$$

(2) Logistics handing agv picking route qualitative factors analysis

In order to reduce the feasible solution set $S$, combined with the qualitative factors in logistics handling agv actual operation, present a genetic algorithm combining qualitative and quantitative, and use it to solve the picking route optimization model. The following logistics handling agv picking route qualitative factors are analyzed.

First of all, considering the logistics handling agv forward direction constraints, namely if the shelves at the agv forward direction is less, the agv should as far as possible toward the horizontal direction of the current, otherwise toward the vertical direction of the current.

Secondly, for agv, every turn will reduce the handling efficiency, so the agv steering factor needs to be considered.

Finally, for the layout of the picking area in the warehouse, the number and area proportion of the shelf and picking platform in warehouse has great influence on the agv picking route.

Above all, the qualitative factors of agv picking route are mainly: (1) the number and the angle of the agv turning, (2)the agv forward direction constraints, (3) the picking warehouse layout.

\section{The improved genetic algorithm combining qualitative and quantitative factors}

Based on "goods to person" logistics handling agv picking mode in rasterized warehouse environment, consider the following three aspects of the traditional genetic algorithm:

1) When environment modeling process, considering the running environment information of agv. 2) When generating the initial population, considering agv less turning and forward direction constraints, artificially generate viable route individual and constitute initial population. 3) In the design of fitness function, considering the route length, the number of the agv turning and the agv forward direction constraints, and it is defined as follows:

The improved genetic algorithm process as shown in Fig. 4. 


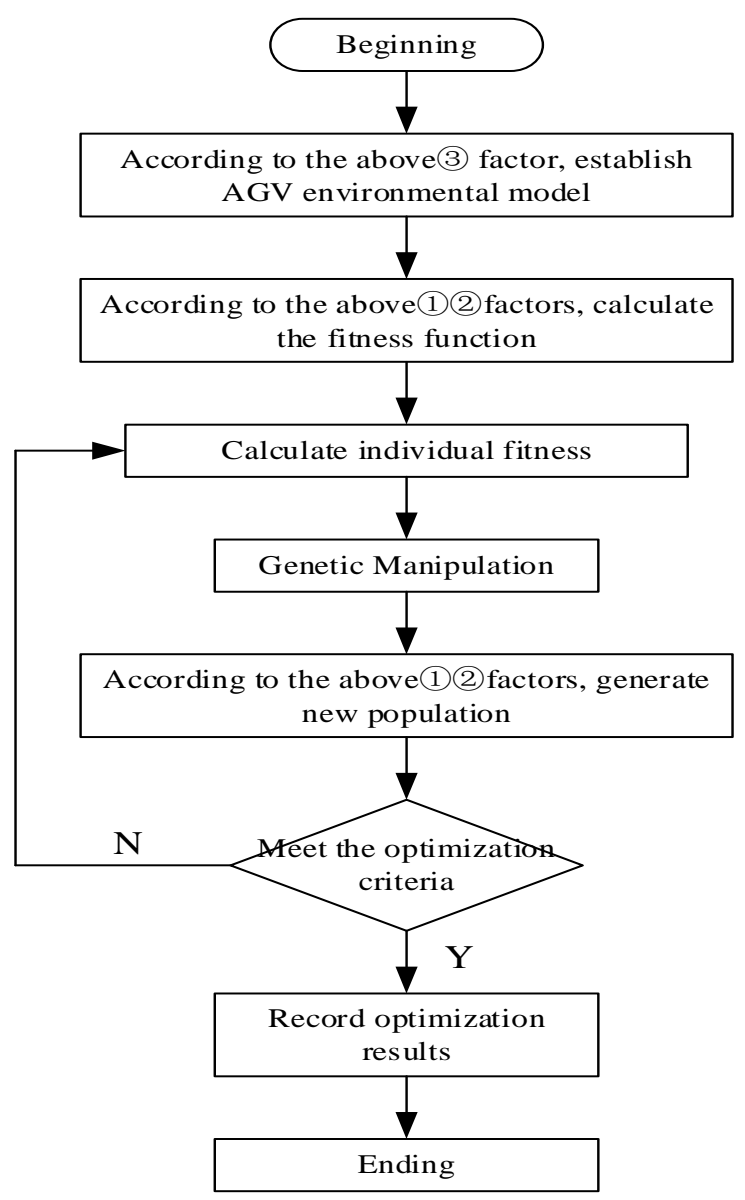

Fig. 4 Improved genetic algorithm flowchart

(1) Individual coding

Every individual is a route from the start grid to the end grid, and encoded on each route. Genetic algorithm usually adopts binary coding, but when using raster numbers coding, the length of the individual is much shorter than the Cartesian coordinates and binary encoding.

Because the agv routes are different, the length of each individual will be different. If the starting grid number is 0 , and the ending grid number is 400 , chromosome is represented by $0 \rightarrow L \rightarrow 400$. In this paper, the extreme value of the route length has been restricted, so it can avoided the situation that chromosome is too long to slow the convergence rate ${ }^{[5]}$.

(2) Generate initial population

Generally, the routes produced by random search are not all feasible routes. In order to increase the convergence speed and computational efficiency, this article considers agv less turning and forward direction constraints, artificially generates initial population, this gives the routes are feasible routes, thereby reducing the aimless characteristics when randomly generated initial population.

(3) Definition of fitness function

Fitness function designed in this paper considers the route length, the number of the agv turning and the agv forward direction constraints, and it is defined as follows:

$$
\begin{aligned}
& f=\frac{1}{\left(1+\frac{1}{\sqrt{n-1}}\right) D+\omega_{p} r(p)}+S(n) \\
& d(p)=\left(1+\frac{1}{\sqrt{n-1}}\right) D
\end{aligned}
$$

$r(p)$ is turning penalty function, $\omega_{p}$ is weighting factor of the penalty function. $d(p)$ is route length function, and $n$ is the sum of all the grids that the agv traveled, $D$ is the sum of all the distance between adjacent grids that the agv traveled, and $S(n)$ is the agv forward direction.

(4) Genetic Manipulation 
1) Selection

Roulette wheel selection is the simplest and most common selection method, and this paper uses this selection policy.

2) Crossover

This paper uses single-point crossover. If two routes have the same grids in addition to the starting point and end point, randomly select one to do a cross-op. If there are not the same grids, give up crossover. After crossover, a new generation replace its parent, in order to get new population.

3) Mutation

Usually, after performing the mutation operation, it will lead to an result agv routes are not unconnected. To overcome this deficiency, this paper does the mutation operation under the premise to ensure the continuity of the routes ${ }^{[4]}$. Specific process is as follows:

Step 1: Optionally select $M \times P_{m}$ individuals from the population, all the selected individuals will be used to perform the mutation, mutation probability is $P_{m}$.

Step 2: Delete the most middle grid of the selected individual, then the previous chromosome individual are divided into front and rear two parts.

Step 3: Make the tail grid of the front part route as the starting point, and the first grid of the rear part route as the end point. Using this kind of method to find a new route, and the separate two parts of the front and rear routes consist a route, this route is continuous. And make it as a new individual of the next population.

4) Delete

Delete operator is used to remove redundant points in the route, its operation is: in the existing routes, delete all the points and the latter repeat point. This can improve processing speed.

(5) Algorithm termination condition

Termination condition is the standard to determine whether the evolutionary algorithm should be the end. Generally set desired goals and the number of evolution generations for the end condition. The improved genetic algorithm in this paper is set to 300 evolution generations. When the algorithm reaches 300 iterations or all of the best individuals remain the same in 50 consecutive generation, the algorithm ends.

(6) Steps of the improved genetic algorithm

Step 1: Initialization population evolutionary generation $t, t=0$.

Step 2: Using raster number (natural number) to code chromosome.

Step 3: Generate the initial population $P(0)=P\left\{p_{1}, p_{2}, \ldots \ldots, p_{M}\right\}$.

Step 4: Calculate every individual's fitness value $f_{i}(i=1,2, \ldots \ldots, M)$.

Step 5: Every individual perform the genetic manipulation in accordance with its own conditions.

Step 6: Record and store operation results.

Step 7: Set the maximum evolution generation $T$ as the termination condition.

Step 8: if this generation groups meet the termination condition, then the algorithm executes the step 9, otherwise backs to step 4.

Step 9: Output the individual coding and the individual's fitness value is the largest.

Step 10: Decoding, obtain the best route.

\section{Simulation results and analysis}

Use matlab7.11 to do Simulation experiments, the set of the various parameters are shown in Table 1 . The grid map size is set to $20 \times 20$. The starting grid number is 1 , the target grid number is 250.

Table 1 Parameters setting table

\begin{tabular}{c|c|c|c}
\hline Population size $M$ & Max evolution $T$ & Crossover probability $P_{c}$ & Mutation probability $P_{m}$ \\
\hline 300 & 100 & 0.8 & 0.2 \\
\hline
\end{tabular}




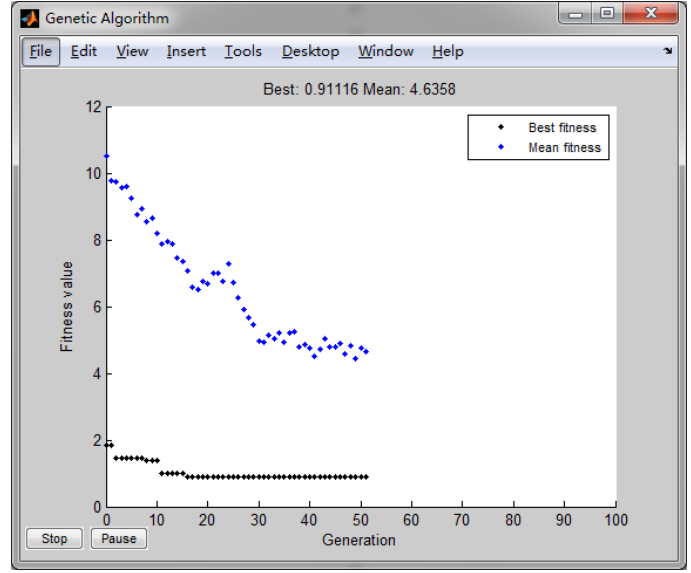

(a) The relationship between evolution generation and fitness value

Fig. 5 Simulation process and results

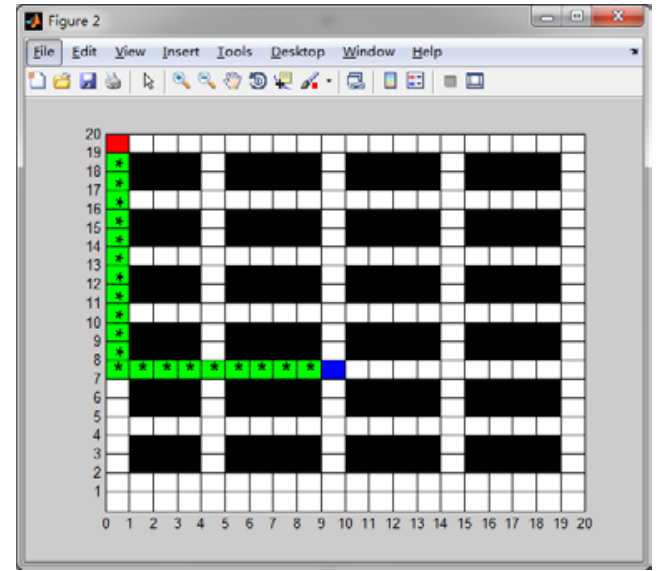

(b) Optimal Path

As Fig. 5 (b) shows, the optimized route is: $1 \rightarrow 21 \rightarrow 41 \rightarrow 61 \rightarrow 81 \rightarrow 101 \rightarrow 121 \rightarrow 141 \rightarrow$ $161 \rightarrow 181 \rightarrow 201 \rightarrow 221 \rightarrow 241 \rightarrow 242 \rightarrow 243 \rightarrow 244 \rightarrow 245 \rightarrow 246 \rightarrow 247 \rightarrow 248 \rightarrow 249 \rightarrow$ 250 , the route length is 22 . As can be seen from the results, the improved genetic algorithm combining qualitative and quantitative factors can find the optimal route.

\section{Conclusions}

According to the simulation results, it is feasible and effective that using the improved genetic algorithm to optimize the agv picking route. But a lot of qualitative factors in the picking system, especially the warehouse layout and the proportion of the shelves in the warehouse, have great influence on the algorithm, so the algorithm is need to be further studied and improved.

\section{Acknowledgements}

This paper is supported by the Funding Project for Technology Key Project of Municipal Education Commission of Beijing (ID:TSJHG201310037036); Funding Project for Beijing key laboratory of intelligent logistics system; Funding Project of Construction of Innovative Teams and Teacher Career Development for Universities and Colleges Under Beijing Municipality (ID:IDHT20130517), and Beijing Municipal Science and Technology Project (ID:Z131100005413004);Funding Project for Beijing philosophy and social science research base specially commissioned project planning (ID:13JDJGD013);Funding Project for Beijing Intelligent Logistics System Collaborative Innovation Center.

\section{Reference}

[1] Li Tianxu, Chen Guangda. Path planning of indoor mobile robot based on improved genetic algorithm[J]. Manufacturing automation,2015,20:31-35.

[2] De Jong H1, Geiselmann J, Hernandez C, Page M. Genetic Network Analyzer: qualitative simulation of genetic regulatory networks [J]. Bioinformatics, 2003, 19(03):336-344.

[3] Shi Tailu. Research and implementation of path planning for single agv in automated warehouse[D]. Anhui University Of Science And Technology,2015.

[4] Jiang Yingjie, Lv Xueqin, Duan Liwei. Path planning of substation inspection robot based on grid genetic algorithm[J]. Science innovation,2015(06):12-14.

[5] Zhao Yuan. Research on path planning of mobile robot based on genetic algorithm[D]. Henan University of Science and Technology,2014.

[6] LiuGuangfeng. Qualitative simulation research based on quantitative information[D]. University of South China,2008. 